\title{
DAC FOR HIGH SPEED AND LOW POWER APPLICATIONS USING ABACUS
}

\author{
Shankarayya G. Kambalimath \\ Department of Electronics and Communication Engineering, \\ Basaveshwar Engineering College, Bagalkot - 587 102, Karnataka, India \\ kambalimathsgerediffmail.com
}

\begin{abstract}
This paper proposes a Chinese Abacus Digital-to-Analog Converter (DAC) for high speed and low power applications like audio and video applications. This circuit of DAC uses resister strings to get a good analog output. The designed DAC uses the algorithm of abacus. Instead of using binary code, here we use abacus code to control the switches. So the complexity and the area will be reduced automatically. The 8-bit DAC is comprised of 12 resistors and 24 NMOS switches. The 8-bit Abacus resistor DAC requires 12 resistors and 24 switches. The 8-bit resistor-string DAC requires 255 resistors and 256 switches. The most important advantages are that the numbers of both resistors and switches are all reduced effectively. The simulation environment uses 1 um process technology.
\end{abstract}

\section{KEYWORDS}

8-bit DAC, Chinese abacus \& DAC circuit

\section{INTRODUCTION}

Every real time signals are analog in nature, as more products perform calculations in the digital or discrete time domain, a device is required to convert from analog to digital known as ADC, after processing it should be in turn converted into analog as human beings can understand only analog signals and the device is digital to analog converter as DAC. Thus Data converters play an important role in an ever-increasing digital world [1]-[3].

Probably the most popular digital-to-analog converter application is converting stored digital audio and/or video signals. For example, stored digital information in MP3 format can be converted into music via a high-precision DAC. Most modern audio signals are stored in digital form (for example MP3s and CDs) and in order to be heard through speakers they must be converted into an analog signal. DACs are therefore founding applications in CD players, digital music players, and PC sound cards [4].

Specialist stand alone DACs can also be found in high-end hi-fi systems. This type normally take the digital output of a compatible CD player and convert the signal into an analog line level output that can then be fed into an amplifier to drive speakers. Similar type of digital-to-analog converters can be found in digital speakers such as USB speakers, and in sound cards. VOIP (Voice over IP) Phone, data transmission over the Internet is done digitally so in order for voice to be transmitted it must be converted to digital using an Analog to Digital computers and be converted into analog again using a DAC so the voice it can be heard on the other end [5]-[9].

David C. Wyld et al. (Eds) : CCSIT, SIPP, AISC, PDCTA, NLP - 2014

pp. 267-274, 2014. (C) CS \& IT-CSCP 2014

DOI : $10.5121 /$ csit.2014.4222 
This paper proposes an 8-bit Abacus resistor DAC. It is a modified version of resistor string DAC where instead of using Binary code to control switches here we are using Abacus code to do the same. So the complexity and area will be reduced. The 8-bit Abacus resistor DAC requires 12 resistors and 24 switches. The 8-bit resistor-string DAC requires 255 resistors and 256 switches. The most important advantages are that the numbers of both resistors and switches are all reduced effectively.

Rest of the paper is organized as follows. Section 2 introduces the operation of Chinese abacus, and binary to abacus module. The proposed Abacus DAC architecture is presented in section 3 . Section 4 explains the result analysis. Section 5 concludes our paper.

\section{CHINESE ABACUS}

The abacus also called a counting frame is a calculating tool that was in use centuries before the adoption of the written modern numeral system and is still widely used by merchants, traders and clerks in Asia, Africa, and elsewhere. Today, abaci are often constructed as a bamboo frame with beads sliding on wires, but originally they were beans or stones moved in grooves in sand or on tablets of wood, stone, or metal. The user of an abacus is called an abacist.

The Chinese abacus is typically $20 \mathrm{~cm}$ ( 8 in) tall and comes in various widths depending on the operator. It usually has more than seven rods. There are two beads on each rod in the upper deck and five beads each in the bottom for both decimal and hexadecimal computation. The suanpan can be reset to the starting position instantly by a quick jerk along the horizontal axis to spin all the beads away from the horizontal beam at the center. Suanpans can be used for functions other than counting. Unlike the simple counting board used in elementary schools, very efficient suanpan techniques have been developed to do multiplication, division, addition, subtraction, square root and cube root operations at high speed.

The binary abacus is used to explain how computers manipulate numbers. The abacus shows how numbers, letters, and signs can be stored in a binary system on a computer, or via ASCII. The device consists of a series of beads on parallel wires arranged in three separate rows. The beads represent a switch on the computer in either an 'on' or 'off' position.

\section{ABACUS DAC ARCHITECTURE}

The proposed Abacus DAC mainly consists of Binary to Abacus conversion block, Switch and Resistor block and Current source.

\subsection{Binary to Abacus conversion}

The designed DAC uses the algorithm of abacus. It uses Abacus code to control switches. Abacus is a tool used for mathematical manipulations. This Abacus tool can also be used to perform coding. The Abacus is made up of a set of unity elements representing the various decades of decimal numbers. 

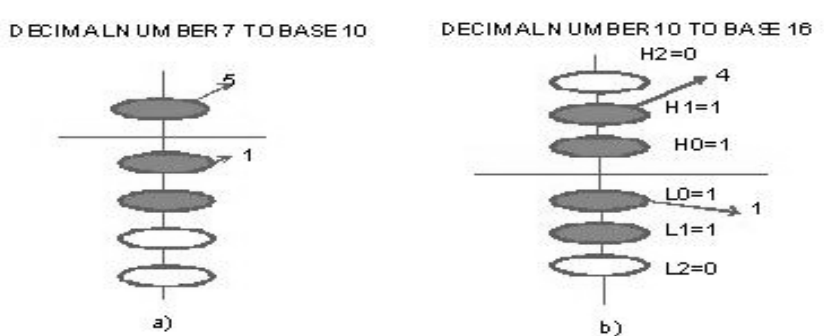

Figure 1. Abacus mapping

A basic column element of the abacus is depicted in figure 1 (a). The configuration represents the number seven. Each column element has one higher bead with a weight of five and four lower beads with a weight of one. In the 8-bit DAC design, each basic column element of this abacus scheme has three lower beads with a weight of one and three higher beads with a weight of four as shown in figure 1 (b). The basic element is able to represent decimal numbers in the range from 0 to 15 as depicted in figure 1 (b). Each weight value of the upper bead is four, and the value of lower bead is one. The binary to Abacus mapping table is as shown in the table 1 .

Table 1 Binary to abacus mapping table

\begin{tabular}{|c|c|c|c|c|c|c|c|c|c|}
\hline \multicolumn{4}{|c|}{ Binary Code } & \multicolumn{6}{|c|}{ Abacus Code } \\
\hline B3 & B2 & B1 & B0 & H2 & H1 & H0 & L2 & L1 & LO \\
\hline 0 & 0 & 0 & 0 & 0 & 0 & 0 & 0 & 0 & 0 \\
\hline 0 & 0 & 0 & 1 & 0 & 0 & 0 & 0 & 0 & 1 \\
\hline 0 & 0 & 1 & 0 & 0 & 0 & 0 & 0 & 1 & 1 \\
\hline 0 & 0 & 1 & 1 & 0 & 0 & 0 & 1 & 1 & 1 \\
\hline 0 & 1 & 0 & 0 & 0 & 0 & 1 & 0 & 0 & 0 \\
\hline 0 & 1 & 0 & 1 & 0 & 0 & 1 & 0 & 0 & 1 \\
\hline 0 & 1 & 1 & 0 & 0 & 0 & 1 & 0 & 1 & 1 \\
\hline 0 & 1 & 1 & 1 & 0 & 0 & 1 & 1 & 1 & 1 \\
\hline 1 & 0 & 0 & 0 & 0 & 1 & 1 & 0 & 0 & 0 \\
\hline 1 & 0 & 0 & 1 & 0 & 1 & 1 & 0 & 0 & 1 \\
\hline 1 & 0 & 1 & 0 & 0 & 1 & 1 & 0 & 1 & 1 \\
\hline 1 & 0 & 1 & 1 & 0 & 1 & 1 & 1 & 1 & 1 \\
\hline 1 & 1 & 0 & 0 & 1 & 1 & 1 & 0 & 0 & 0 \\
\hline 1 & 1 & 0 & 1 & 1 & 1 & 1 & 0 & 0 & 1 \\
\hline 1 & 1 & 1 & 0 & 1 & 1 & 1 & 0 & 1 & 1 \\
\hline 1 & 1 & 1 & 1 & 1 & 1 & 1 & 1 & 1 & 1 \\
\hline
\end{tabular}




\subsection{Switch and resistor design}

The Proposed DAC consists of switch and resistor block, which is as shown in figure 2. When digital input is 1, the circuit works on principle, to let to SW1 is ON, SW2 is OFF. The current pass through to be composed by the NMOS switch and resistor. Thus it contributes some voltage at output. When digital input is $0, \mathrm{SW} 1$ is OFF, SW2 is ON. The current pass through to be composed by the NMOS switch. Thus voltage contributed will be zero. The 8-bit DAC has 12 Switch and Resistor blocks, and completely series 12 switch and resistor circuit blocks.

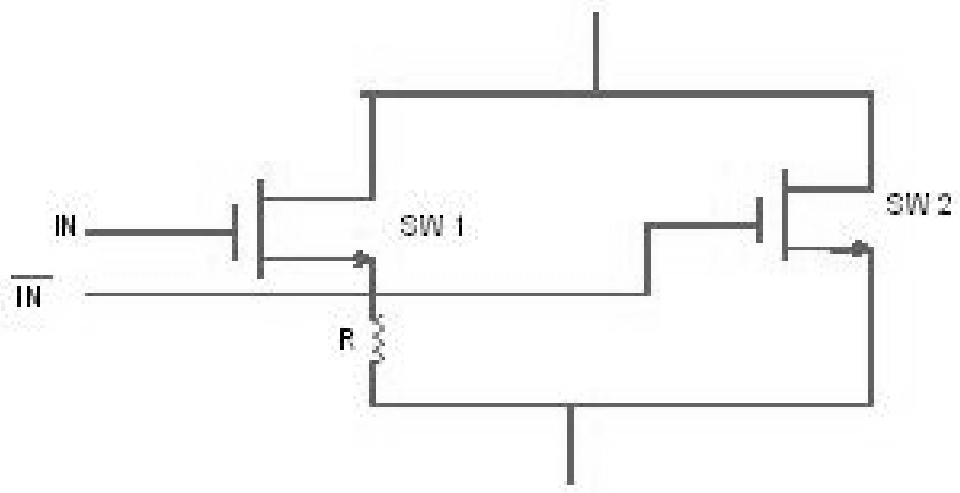

Figure 2. Switch and resistor block

\subsection{Current source}

In Beta multiplier circuit we are adding the resistor at the source side to make it supply independent and combination of NMOS and PMOS devices provide necessary biasing for the current mirror circuit. Figure 3 shows the Beta-multiplier reference for biasing current mirror circuit. In any self-biased circuit the unwanted one where zero current flows in the circuit. This unwanted state occurs when the gates of M1/M2 are at ground while the gates of M3/M4 are at VDD. When in this state, the gate of M5 is at ground and so it is off. The gate of M6 is somewhere between VDD and VDD - VTHP. M7, which behaves like an NMOS switch, turns on and leaks current into the gates of M1/M2 from the gates of M3/M4.

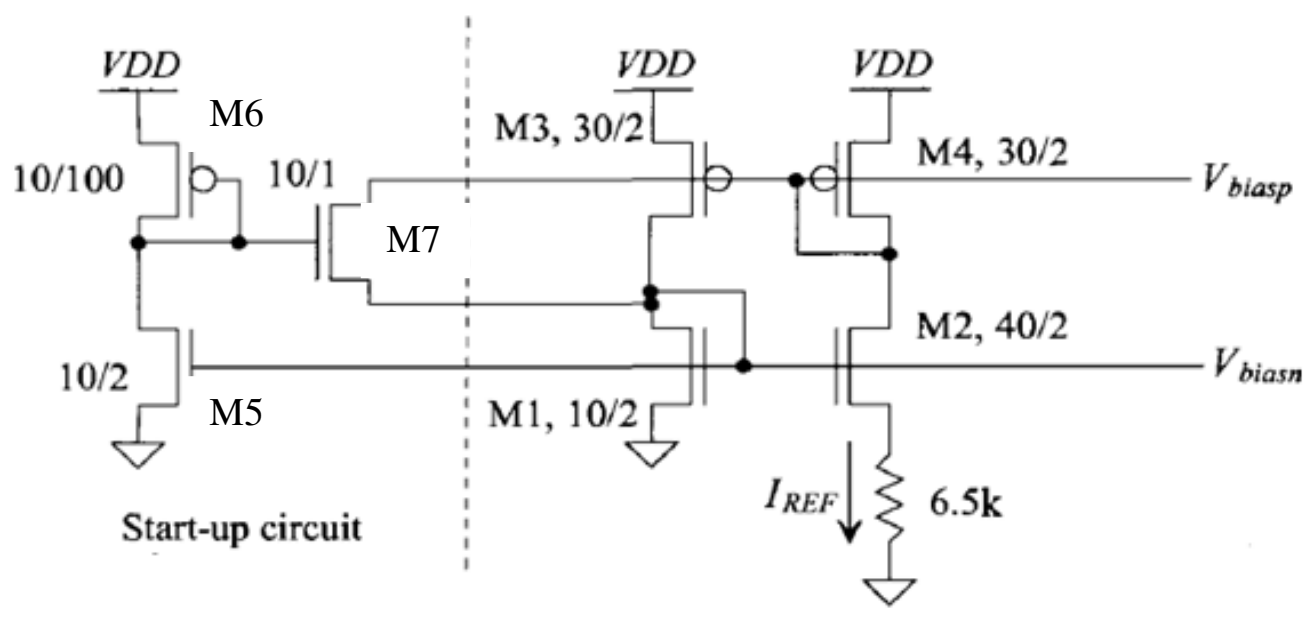

Figure 3. Beta-multiplier reference for biasing 
This causes the current to snap to the desired state and M7 to turn off. Note that during normal operation the start-up circuit should not affect the Beta-multiplier's operation. The current through M7 should be zero (or very small). Finally the proposed Abacus DAC consists of 12 switch and resistor blocks, two binary to Abacus mapping blocks and a current sources. 8-bit binary inputs from B0 to B7 are given as input to binary to Abacus conversion block which generates corresponding Abacus code. These generated Abacus code are in turn given to switch and resistor block to control the switches. Whenever Abacus code is 1 the respective switch and resistor block will contribute voltage at the output. The overall voltage will be sum of the voltage contributed by all 12 switch and resistor block. Thus digital domain is converted in to analog voltage.

\section{RESULT ANALYSIS}

To test the performance effectiveness of the proposed scheme, some of the simulation results analyzed are as follows: ideal 4-bit Abacus DAC output shown in figure 4, ideal 8-bit Abacus DAC output shown in figure 5, practical 8-bit Abacus DAC output shown in figure 6, differential non-linear error (DNL) is shown in figure 7, integral non-linear error (INL) is shown in figure 8 and offset error is shown in figure 9.

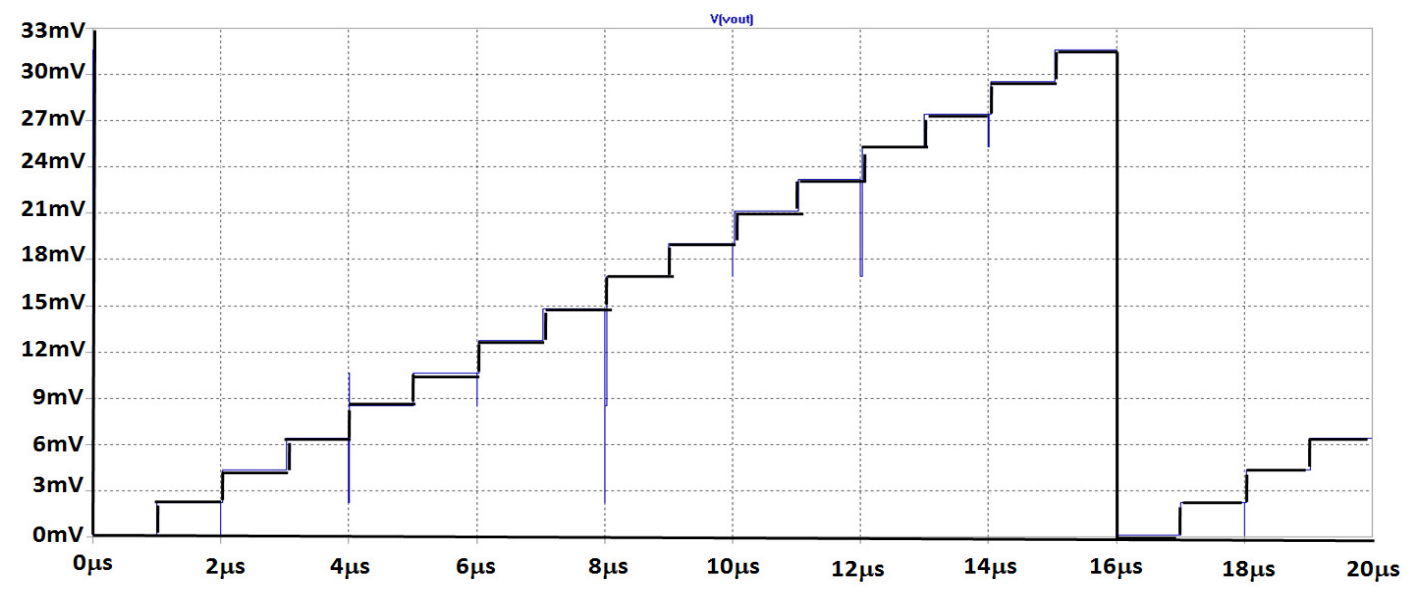

Figure 4. Ideal 4-bit Abacus DAC output

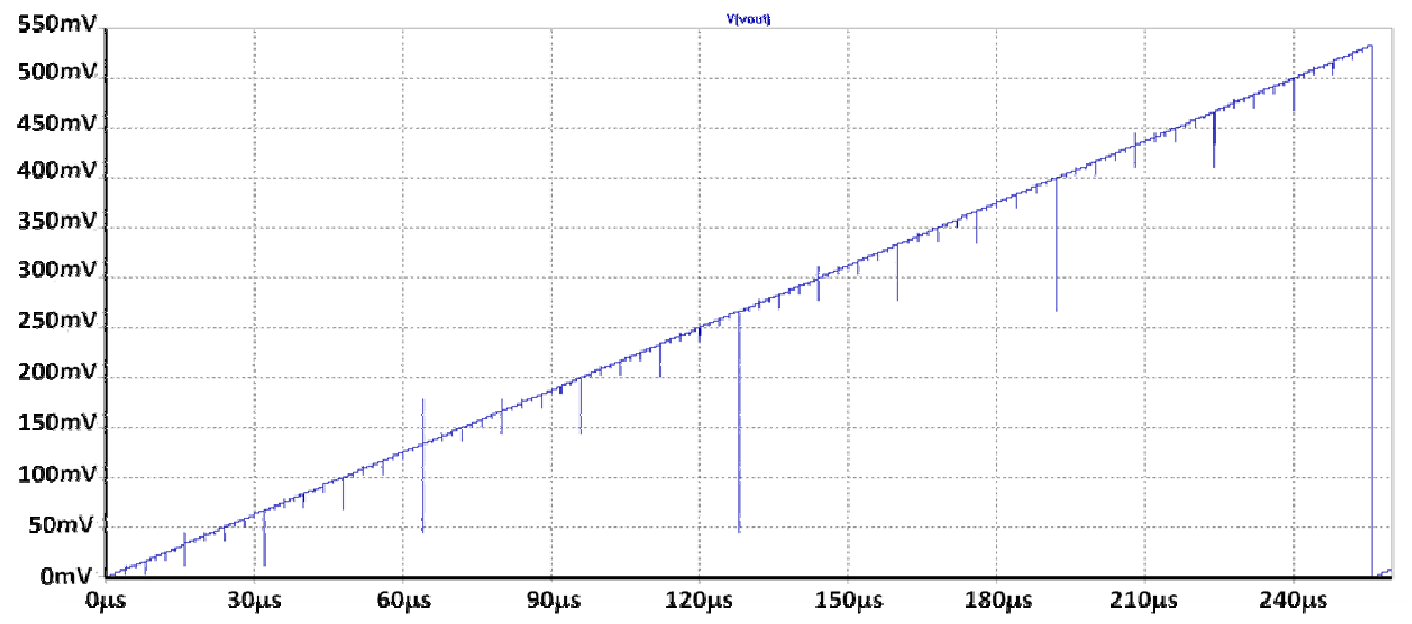

Figure 5. Ideal 8-bit Abacus DAC output 


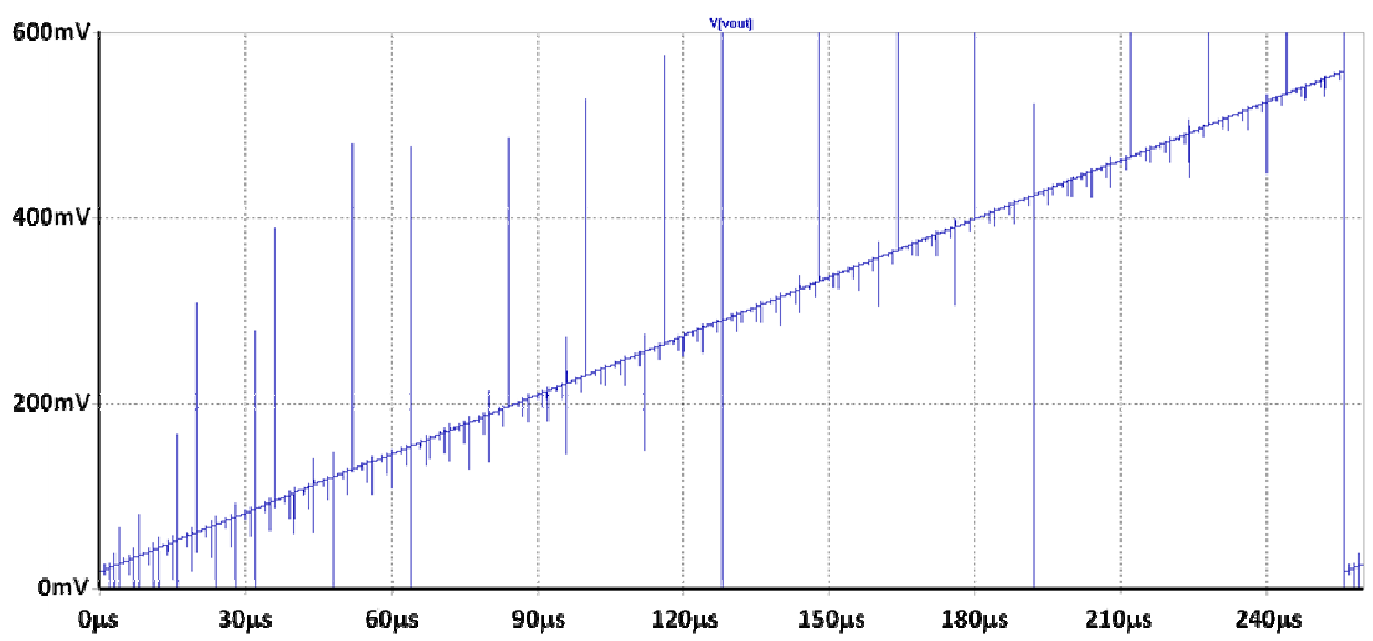

Figure 6. Practical 8-bit Abacus DAC output

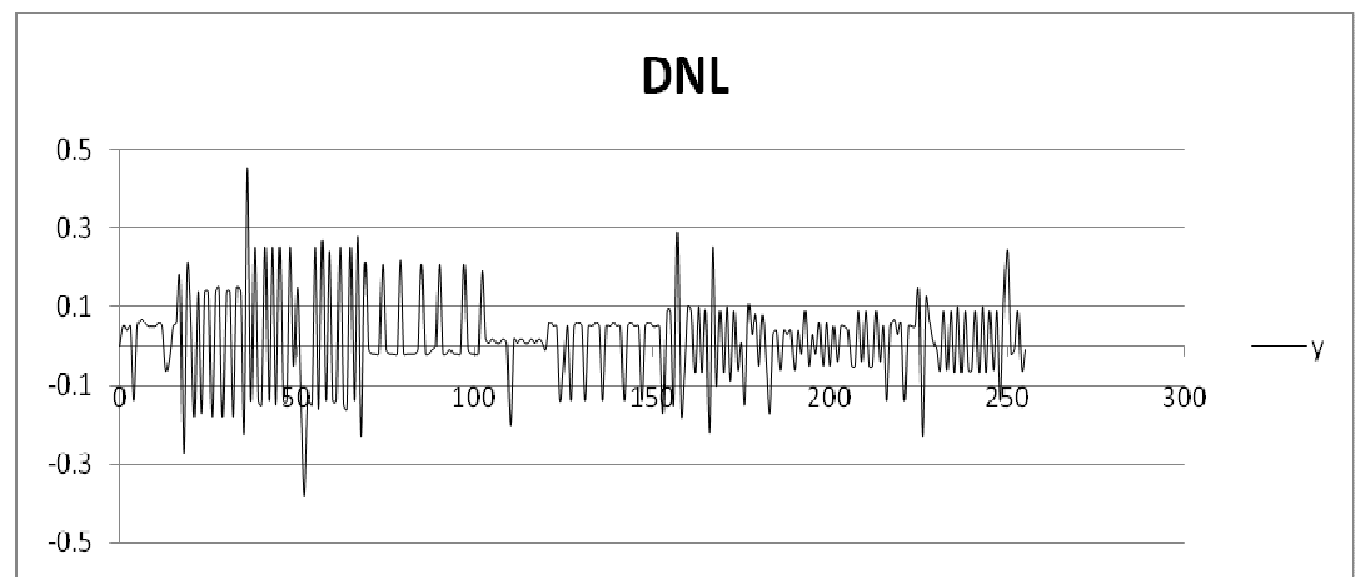

Figure 7. Differential non-linear error (DNL)

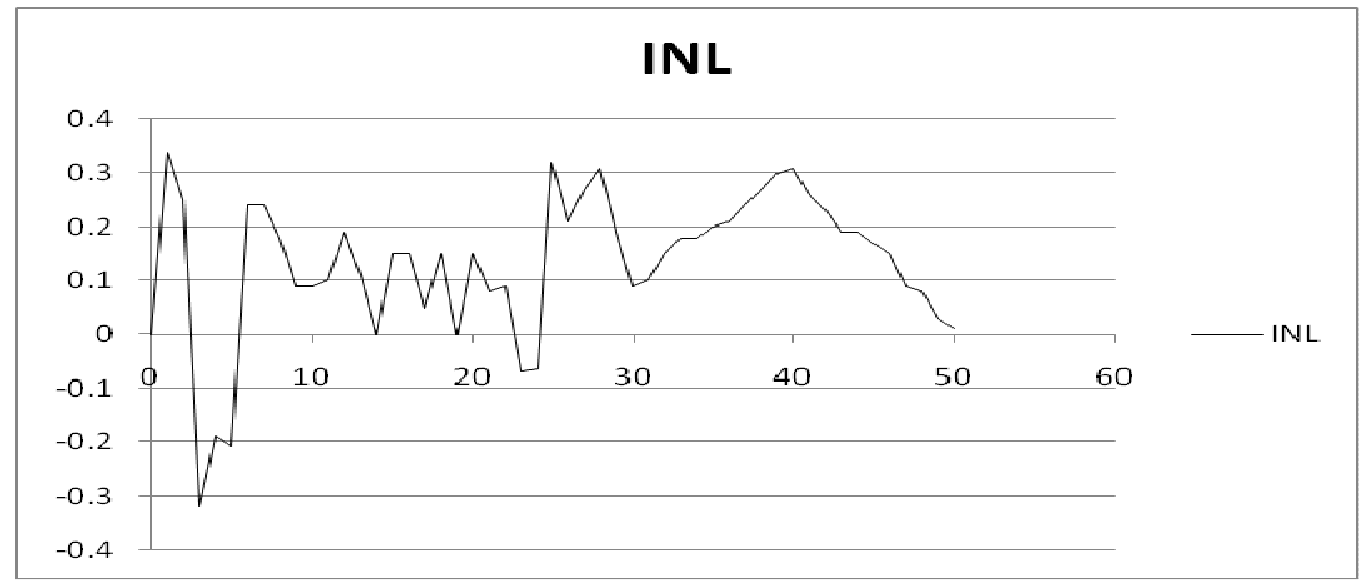

Figure 8. Integral non-linear error (INL) 


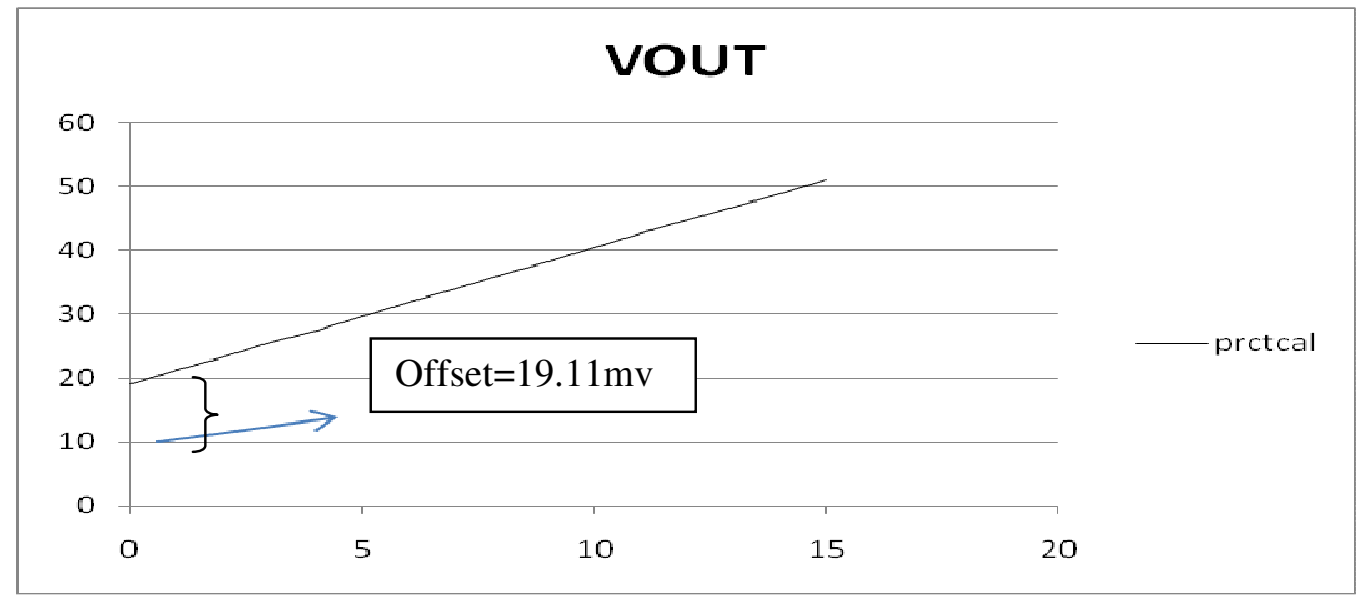

Figure 9. Offset error

\section{CONCLUSIONS}

The paper presents an 8-bit ABACUS digital to analog converter. The abacus algorithm is successfully adopted in the DAC design. Results show that this scheme reduces effectively the number of resistors of conventional resistor string DAC. The 8-bit digital to analog converter has low power consumption. $1 \mu \mathrm{m}$ process technology is used in the implementation of the proposed DAC. The simulation results of DNL and INL are below 0.35 LSB and 0.4 LSB, respectively.

\section{REFERENCES}

[1] Shun-He Huang, Chien-Hung Lin, Shu-Chung Yi and Jin-Jia Chen, "A Chinese Abacus DAC for Video Applications", Proc. Third International Conference on Intelligent Information Hiding and Multimedia Signal Processing (IIHMSP), pp. 507-510, November 2007.

[2] C.-C. Tsai, C.-H. Lai, W.-T. Lee and J.-O. Wu, "10 bit switched-current digital-to-analogue converter”, Proc. IEE Circuits Design Systems, pp. 287-290 June 2005.

[3] Miki, T., Nakamura, Y., Nakaya, M., Asai, S., Akasaka, Y., and Horiba, Y., “An 80-MHz 8-bit CMOS D/A Converter”, IEEE Journal of Solid-State Circuits, Volume 21, Issue 6, Dec 1986, pp. 983-988.

[4] Shu-Chung Yi, Kun-Tse Lee, Jin-Jia Chen, Chien-Hung Lin, Chuen-Ching Wang, Chin-Fa Hsieh, and Chih- Yung Lu, "The new architecture of radix-4 Chinese abacus adder", Proc. IEEE 36th International Symposium on Multiple-Valued Logic, May 2006.

[5] Zi-Yi Zhao, Jian-Hung Lin, Yu-Zhi Xie, Yen-Ju Chen, Yi-Jie Lin, and Shu-Chung Yi., "The novel Chinese abacus adder", Prcc. international symposium on VLSI design automation and test, 25-27 April 2007

[6] Huynh, J., Ngo, B., Pham, M., and He, L, "Design of a 10 Bit TSMC 0.25 $\mu$ m CMOS digital to analog converter", Proc. Sixth International Symposium on Quality Electronic Design, 21-23 March 2005, pp. 187-192.

[7] Moonsik Song, Bongsoon Kang, and Eurho Joe, "A 10-Bit 80MHz 3.0V For Video CMOS D/A Converter Applications", IEEE Transactions on Consumer Electronics, Volume 43, Issue 3, Aug. 1997, pp. 868-872.

[8] Leonard, J., Weste, N., Bodony, L., Harston, S., and Meaney, R., “A 66-MHz DSP augmented RAMDAC for smooth-shaded graphic applications", IEEE Journal of Solid-State Circuits, Volume 26, Issue 3, Mar 1991, pp. 217-228.

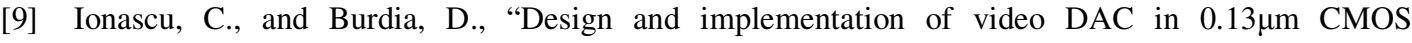
technology", IEEE SCS 2003. International Symposium on Signals, Circuits and Systems, Volume 2, 10-11 July 2003, pp.381-384. 


\section{AUTHOR}

Shankarayya G Kambalimath received the B.E. and M.E. degrees in Electronics and Communication Engineering from the Gulbaraga University and Bangalore University Karnataka, India in the year 1993 and 1999 respectively. He is currently pursuing the Ph.D. degree at the Visvesvaraya Technological University, Belgaum, Karnataka, India. His main research interests include VLSI and signal processing.

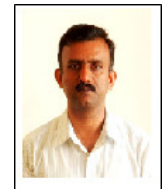

